\title{
A comparison of estimation accuracy by the use of KF, EKF \& UKF filters
}

\author{
S. Konatowski \& A. T. Pieniężny \\ Department of Electronics, Military University of Technology, Poland
}

\begin{abstract}
This paper considers the problem of applying the Kalman filters to nonlinear systems. The Kalman filter (KF) is an optimal linear estimator when the process noise and the measurement noise can be modeled by white Gaussian noise. The KF only utilizes the first two moments of the state (mean and covariance) in its update rule. In situations when the problems are nonlinear or the noise that distorts the signals is non-Gaussian, the Kalman filters provide a solution that may be far from optimal. Nonlinear problems can be solved with the extended Kalman filter (EKF). This filter is based upon the principle of linearization of the state transition matrix and the observation matrix with Taylor series expansions. Exploiting the assumption that all transformations are quasi-linear, the EKF simply makes linear all nonlinear transformations and substitutes Jacobian matrices for the linear transformations in the KF equations. The linearization can lead to poor performance and divergence of the filter for highly non-linear problems. An improvement to the extended Kalman filter is the unscented Kalman filter (UKF). The UKF approximates the probability density resulting from the nonlinear transformation of a random variable. It is done by evaluating the nonlinear function with a minimal set of carefully chosen sample points. The posterior mean and covariance estimated from the sample points are accurate to the second order for any nonlinearity. The paper presents a comparison of the estimation quality for two nonlinear measurement models of the following Kalman filters: covariance filter (KF), extended filter (EKF) and unscented filter (UKF).

Keywords: nonlinear model, discrete Kalman filter, extended Kalman filter, unscented Kalman filter, integrated navigation system.
\end{abstract}




\section{Introduction}

The classical Kalman filter is used for linear dynamic systems [1] moreover extended Kalman filter EKF for nonlinear systems [1, 3] or unscented Kalman filter UKF [2, 4-8]. Unscented Kalman filter with comparison to EKF is not based on linear model but operates on the statistical parameters of the measurement and state vectors that are subsequently nonlinearly transformed. The unscented Kalman filter is based on the unscented transform (UT).

Kalman filtration $[1,3]$ is based on the following models of state and measurement vectors respectively:

$$
\begin{gathered}
\mathbf{x}(k+1)=\mathbf{f}[\mathbf{x}(k), \mathbf{u}(k), \mathbf{w}(k)] \quad \text { for } \quad \mathbf{w}(k) \sim N[\mathbf{0}, \mathbf{Q}(k)], \\
\mathbf{z}(k+1)=\mathbf{h}[\mathbf{x}(k), \mathbf{v}(k)] \quad \text { for } \quad \mathbf{v}(k) \sim N[\mathbf{0}, \mathbf{R}(k)] .
\end{gathered}
$$

Vector $\mathbf{x}(k)$ is $n$-dimensional state vector in the moment $k, \mathbf{z}(k)$ is $p$-dimensional measurement vector in the moment $k, \mathbf{f}(\mathbf{x}, \mathbf{u}, \mathbf{w})$ denotes nonlinear state function describing dynamic behavior of the system between $k+1$ and $k$ moments, $\mathbf{u}$ is the input system vector, $\mathbf{w}$ is the noise state vector, $\mathbf{Q}$ is the covariance matrix of the noise state (denotes uncertainty in the dynamic model during transition from $k+1$ to $k$ moments, $\mathbf{h}(\mathbf{x}, \mathbf{v})$ denotes nonlinear measurement function, $\mathbf{v} p$-dimensional vector of measurement noise, $\mathbf{R}$ is covariance matrix of measurement errors with dimensions $p \times p, \mathbf{P}$ is covariance matrix of state vector with dimensions $n \times n$.

\section{Object dynamics and measurement models}

In this paper process model is described by state vector in the following form:

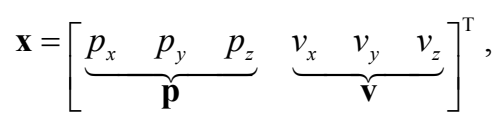

where $\mathbf{p}$ is three-dimensional position vector (in Cartesian coordinates). Vector $\mathbf{v}$ is three-dimensional speed vector.

State matrix has the form:

$$
\mathbf{F}=\left[\begin{array}{cc}
\mathbf{I}_{3 \times 3} & \Delta t \mathbf{I}_{3 \times 3} \\
\mathbf{0}_{3 \times 3} & \mathbf{I}_{3 \times 3}
\end{array}\right],
$$

where $\Delta t$ is the time step between moments $k$ and $k+1, \mathbf{I}$ is identity matrix.

In this case the state vector (2) and state matrix (3) are identical for covariance filter (KF), extended filter (EKF) and unscented filter (UKF).

\subsection{System with constant velocity}

The object moves with constant velocity (acceleration is zero). Velocity components $\left(v_{x, y, z}\right)$ are additive Gaussian noise. Fig. 1 presents analyzed model in the graphic form. 


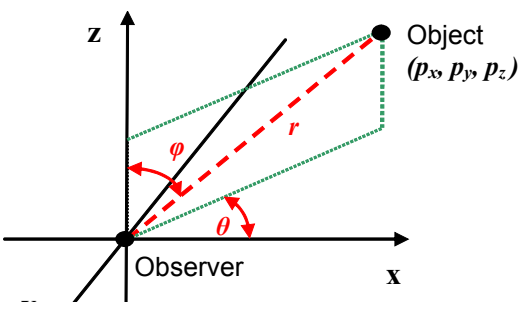

Figure 1: $\quad$ Positioning principle.

The measured parameters in the measurement model are: $r$ - distance from object to observer (radar), $\theta$ - azimuth, $\varphi$ - elevation. Dependency between object position in the Cartesian coordinates and measurements in spherical coordinates is described by nonlinear function. Thus the measurement vector is given by:

$$
\mathbf{z}=[\underbrace{\sqrt{p_{x}^{2}+p_{y}^{2}+p_{z}^{2}}}_{r} \underbrace{\arctan \left(p_{y} / p_{x}\right)}_{\theta} \underbrace{\arccos \left(p_{z} / \sqrt{p_{x}^{2}+p_{y}^{2}+p_{z}^{2}}\right)}_{\varphi}]^{\mathrm{T}} .
$$

Measurement matrix for discrete and unscented filters is given by:

$$
\mathbf{H}=\left[\begin{array}{ll}
\mathbf{I}_{3 \times 3} & \mathbf{0}_{3 \times 3}
\end{array}\right] .
$$

The vector function $\mathbf{h}(*)$ has the following form:

$$
\mathbf{h}=\left[\begin{array}{lll}
r & \theta & \varphi
\end{array}\right]^{\mathrm{T}} .
$$

In the case of extended filter, linearization of the measurement function $\mathbf{h}$ for each measurement step by the use of partial derivative relatively to all elements of state vector should be made. The final measurement matrix for extended Kalman filter is as follows:

$$
\mathbf{H}=\left.\frac{\partial \mathbf{h}}{\partial \mathbf{x}}\right|_{\mathbf{x}=\widehat{\mathbf{x}}(k)}=\left[\begin{array}{cccccc}
p_{x} / r & p_{y} / r & p_{z} / r & 0 & 0 & 0 \\
p_{y} /\left(r^{2}-p_{z}^{2}\right) & p_{x} /\left(r^{2}-p_{z}^{2}\right) & 0 & 0 & 0 & 0 \\
\frac{p_{x} p_{z}}{r^{2} \sqrt{r^{2}-p_{z}^{2}}} & \frac{p_{y} p_{z}}{r^{2} \sqrt{r^{2}-p_{z}^{2}}} & \frac{-\sqrt{r^{2}-p_{z}^{2}}}{r^{2}} & 0 & 0 & 0
\end{array}\right],
$$

where Cartesian object coordinates are given by:

$$
p_{x}=r \cos \theta \sin \varphi, \quad p_{y}=r \sin \theta \cos \varphi, \quad p_{z}=r \cos \varphi .
$$

\subsection{System with complex movement}

As an example of complex dynamics, the object movement around $\boldsymbol{z}$ axis is presented. This situation causes nonlinear relationships both in state and measurement matrix. Figure 2 in detail illustrates considered object. 

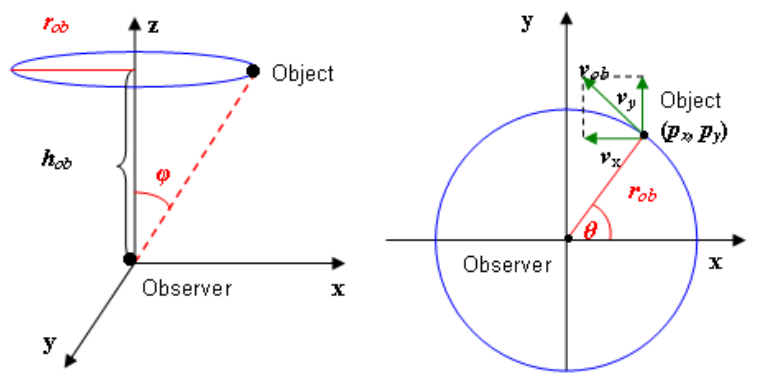

Figure 2: $\quad$ Positioning principle.

One can see that a nonlinear relation exists between measurements from the system and elements of the motion:

$$
\tan \theta=v_{x} / v_{y}, \quad v_{x}=v_{o b} \sin \theta, \quad v_{y}=v_{o b} \cos \theta, \quad v_{o b}=\sqrt{v_{x}^{2}+v_{y}^{2}} .
$$

In the presented system initial values of vector state are described by the followings:

$$
\mathbf{x}(0)=\left[\begin{array}{llllll}
r_{o b} & 0 & h_{o b} & 0 & v_{o b} & 0
\end{array}\right]^{\mathrm{T}} .
$$

Nonlinear state functions (in the moment $k$ ) are defined as follows:

$$
\mathbf{f}(k)=\left[\begin{array}{c}
f_{1} \\
f_{2} \\
f_{3} \\
f_{4} \\
f_{5} \\
f_{6}
\end{array}\right]=\left[\begin{array}{c}
r_{o b} \cos \left[\arctan \left(p_{y} / p_{x}\right)+\omega(k) \Delta t\right] \\
r_{o b} \sin \left[\arctan \left(p_{y} / p_{x}\right)+\omega(k) \Delta t\right] \\
p_{z}(k)+v_{z}(k) \Delta t \\
v_{o b} \sin \left[\arctan \left(p_{y} / p_{x}\right)+\omega(k) \Delta t\right] \\
v_{o b} \cos \left[\arctan \left(p_{y} / p_{x}\right)+\omega(k) \Delta t\right] \\
v_{z}(k)
\end{array}\right],
$$

where the azimuth and the angular velocity of the object can be calculated via the following formulas:

$$
\theta(k+1)=\theta(k)+\omega(k) \Delta t, \quad \omega(k)=v_{o b}(k) / r_{o b}(k) \text { for } r_{o b}=\sqrt{p_{x}^{2}+p_{y}^{2}} .
$$

This nonlinear equation requires linearization, which in extended Kalman filter is performed around the estimated object's trajectory. For the EKF state matrix has been calculated as a matrix of derivatives of nonlinear $\mathbf{f}\left({ }^{*}\right)$ function with respect to the components of the state vector $\mathbf{x}$.

$$
\mathbf{F}=\partial \mathbf{f} / \partial \mathbf{x},
$$

where: 


$$
\begin{aligned}
& \frac{\partial f_{1}}{\partial p_{x}}=\frac{p_{x}}{r_{o b}} \cos \gamma+r_{o b}\left(\frac{v_{o b} \Delta t p_{x}}{r_{o b}^{3}}+\frac{p_{y}}{p_{x}^{2} \beta}\right) \sin \gamma, \\
& \frac{\partial f_{1}}{\partial p_{y}}=\frac{p_{y}}{r_{o b}} \cos \gamma+r_{o b}\left(\frac{v_{o b} \Delta t p_{y}}{r_{o b}^{3}}-\frac{1}{p_{x} \beta}\right) \sin \gamma, \\
& \frac{\partial f_{1}}{\partial p_{z}}=0, \frac{\partial f_{1}}{\partial v_{x}}=\frac{\Delta t v_{x}}{v_{o b}} \sin \gamma, \frac{\partial f_{1}}{\partial v_{y}}=\frac{\Delta t v_{y}}{v_{o b}} \sin \gamma, \frac{\partial f_{1}}{\partial v_{z}}=0, \\
& \frac{\partial f_{2}}{\partial p_{x}}=\frac{p_{x}}{r_{o b}} \sin \gamma-r_{o b}\left(\frac{p_{y}}{p_{x}^{2} \beta}+\frac{v_{o b} \Delta t p_{x}}{r_{o b}^{3}}\right) \cos \gamma, \\
& \frac{\partial f_{2}}{\partial p_{y}}=\frac{p_{y}}{r_{o b}} \sin \gamma+r_{o b}\left(\frac{1}{p_{x} \beta}-\frac{v_{o b} \Delta t p_{y}}{r_{o b}^{3}}\right) \cos \gamma, \\
& \frac{\partial f_{2}}{\partial p_{z}}=0, \quad \frac{\partial f_{2}}{\partial v_{x}}=\frac{\Delta t v_{x}}{v_{o b}} \cos \gamma, \frac{\partial f_{2}}{\partial v_{y}}=\frac{\Delta t v_{y}}{v_{o b}} \cos \gamma, \frac{\partial f_{2}}{\partial v_{z}}=0, \\
& \frac{\partial f_{3}}{\partial p_{x}}=0, \frac{\partial f_{3}}{\partial p_{y}}=0, \quad \frac{\partial f_{3}}{\partial p_{z}}=1, \quad \frac{\partial f_{3}}{\partial v_{x}}=0, \frac{\partial f_{3}}{\partial v_{y}}=0, \frac{\partial f_{3}}{\partial v_{z}}=\Delta t, \\
& \frac{\partial f_{4}}{\partial p_{x}}=v_{o b}\left(-\frac{p_{y}}{p_{x}^{2} \beta}-\frac{v_{o b} \Delta t p_{x}}{r_{o b}^{3}}\right) \cos \gamma, \frac{\partial f_{4}}{\partial p_{y}}=v_{o b}\left(\frac{1}{p_{x} \beta}-\frac{v_{o b} \Delta t p_{y}}{r_{o b}^{3}}\right) \cos \gamma, \\
& \frac{\partial f_{4}}{\partial p_{z}}=0, \quad \frac{\partial f_{4}}{\partial v_{x}}=\frac{\left(r_{o b} \sin \gamma+v_{o b} \Delta t \cos \gamma\right)}{r_{o b} v_{o b}} v_{x} \\
& \frac{\partial f_{4}}{\partial v_{y}}=\frac{\left(r_{o b} \sin \gamma+v_{o b} \Delta t \cos \gamma\right)}{r_{o b} v_{o b}} v_{y}, \quad \frac{\partial f_{4}}{\partial v_{z}}=0, \\
& \frac{\partial f_{5}}{\partial p_{x}}=v_{o b}\left(\frac{p_{y}}{p_{x}^{2} \beta}+\frac{v_{o b} \Delta t p_{x}}{r_{o b}^{3}}\right) \sin \gamma, \frac{\partial f_{5}}{\partial p_{y}}=v_{o b}\left(\frac{v_{o b} \Delta t p_{y}}{r_{o b}^{3}}-\frac{1}{p_{x} \beta}\right) \cos \gamma, \\
& \frac{\partial f_{5}}{\partial p_{z}}=0, \quad \frac{\partial f_{5}}{\partial v_{x}}=\frac{\left(r_{o b} \cos \gamma-v_{o b} \Delta t \sin \gamma\right)}{r_{o b} v_{o b}} v_{x} \\
& \frac{\partial f_{5}}{\partial v_{y}}=\frac{\left(r_{o b} \cos \gamma-v_{o b} \Delta t \sin \gamma\right)}{r_{o b} v_{o b}} v_{y}, \quad \frac{\partial f_{5}}{\partial v_{z}}=0, \\
& \frac{\partial f_{6}}{\partial p_{x}}=0, \frac{\partial f_{6}}{\partial p_{y}}=0, \frac{\partial f_{6}}{\partial p_{z}}=0, \quad \frac{\partial f_{6}}{\partial v_{x}}=0, \quad \frac{\partial f_{6}}{\partial v_{y}}=0, \frac{\partial f_{6}}{\partial v_{z}}=1,
\end{aligned}
$$

for: $\gamma=\arctan \left(p_{y} / p_{x}\right)+\Delta t v_{o b} / r_{o b}, \quad \beta=1+p_{y}^{2} / p_{x}^{2}$.

For discrete and unscented Kalman filter state matrix has a form given by (3). An observation matrix $\mathbf{H}$ in the measurement model is identical as in the first model (11). 


\section{Simulation results}

The accuracy comparisons have been examined by the use of simulation in the Matlab environment. In order to ensure the same conditions, research of filters were realized with identical form of state vector covariance matrix $\mathbf{Q}$, measurement vector $\mathbf{R}$ and initial state vector covariance matrix $\mathbf{P}(0)$ in both systems. Similarly to Julier et al [4] the following parameters of unscented transform have been assumed: $\lambda=3, \beta=1, \kappa=3$.

Furthermore the following values of noise covariance matrix have been applied:

$$
\mathbf{Q}=\left[\begin{array}{cc}
\operatorname{diag}\left[0.0225 \mathrm{~m}^{2}\right]_{3 \times 3} & \mathbf{0}_{3 \times 3} \\
\mathbf{0}_{3 \times 3} & \operatorname{diag}\left[0.49 \mathrm{~m}^{2} \mathrm{~s}^{-2}\right]_{3 \times 3}
\end{array}\right],
$$

covariance matrix of measurement noise:

$$
\mathbf{R}=\operatorname{diag}\left[\begin{array}{lll}
0.7225 \mathrm{~m}^{2} & 0.16 \mathrm{deg}^{2} \quad 0.16 \mathrm{deg}^{2}
\end{array}\right]
$$

initial covariance matrix of vector state errors:

$$
\mathbf{P}(0)=\left[\begin{array}{cc}
\operatorname{diag}\left[1 \mathrm{~m}^{2}\right]_{3 \times 3} & \mathbf{0}_{3 \times 3} \\
\mathbf{0}_{3 \times 3} & \operatorname{diag}\left[1 \mathrm{~m}^{2} \mathrm{~s}^{-2}\right]_{3 \times 3}
\end{array}\right] .
$$

\subsection{Examination of a system with constant velocity}

Examination results are presented for covariance filter (DKF - green line), extended filter (EKF - red line) and for unscented filter (UKF - blue line). The examinations results include values of mean square error (mse) of estimated state vector:

$$
\text { mse } * \mathrm{KF}=\sqrt{\left(\hat{\mathbf{x}}-\mathbf{x}_{\text {real }}\right)^{\mathrm{T}}\left(\hat{\mathbf{x}}-\mathbf{x}_{\text {real }}\right) / n},
$$

and covariance error $\mathbf{P}$, according to Kalman filtering theory, estimated component of state vector $\mathbf{x}$ (Fig. 3-5):

$$
\operatorname{cov} * \mathrm{KF}=\mathbf{P}(k+1)=\mathbf{F}(k) \mathbf{P}(k) \mathbf{F}(k)^{\mathrm{T}}+\mathbf{Q}(k) .
$$

Mean square error and covariance error of speed components (Fig. 6-8) has also been done.

\subsection{Examination of a system with complex movement}

The system examination conditions are the same as for system with constant velocity. Results for covariance filter DKF, extended filter EKF and unscented filter UKF are presented. They include values of mse error of estimated state vector and covariance error estimated components of the position (Fig. 9-11) and values of mse error and covariance error of speed components (Fig. 12-14). 


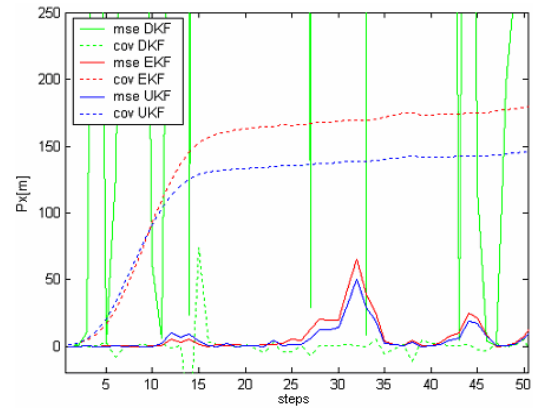

Figure 3: Mean square error of the estimated component $p_{x}$ and covariance error $P_{p x}$ of the position.

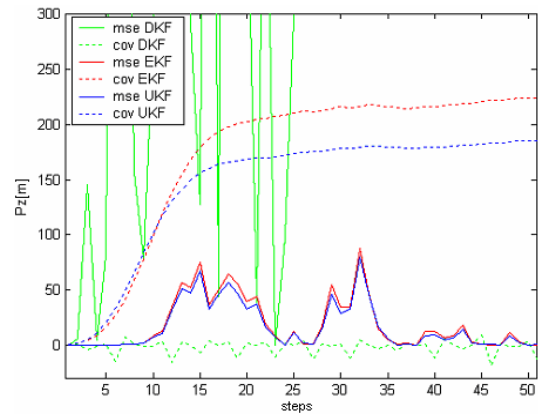

Figure 5: Mean square error of the estimated component $p_{z}$ and covariance error $P_{p z}$ of the position.

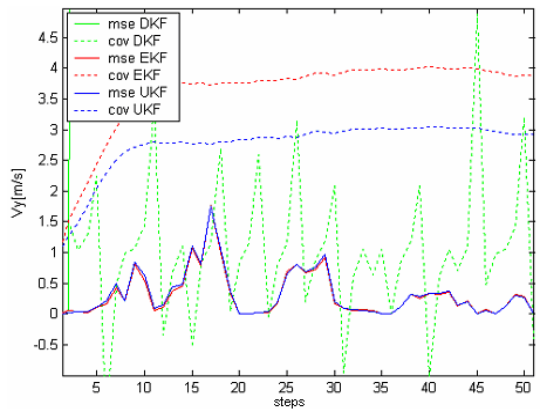

Figure 7: Mean square error of estimated $v_{y}$ and covariance error $P_{v y}$ of speed component.

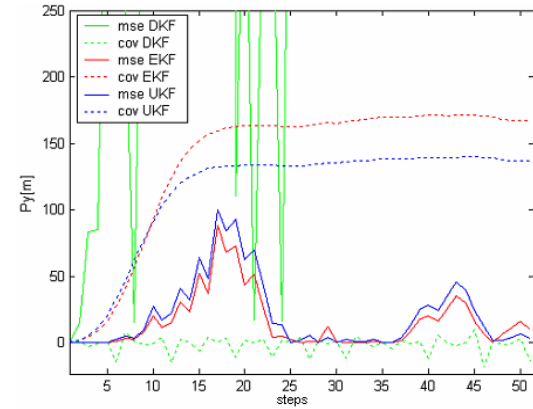

Figure 4: Mean square error of the estimated component $p_{y}$ and covariance error $P_{p y}$ of the position.

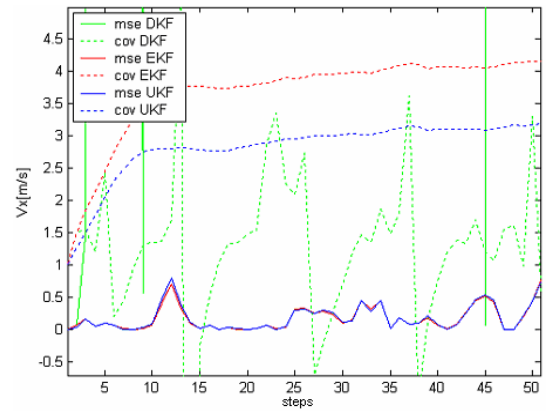

Figure 6: Mean square error of estimated $v_{x}$ and covariance error $P_{v x}$ of speed component

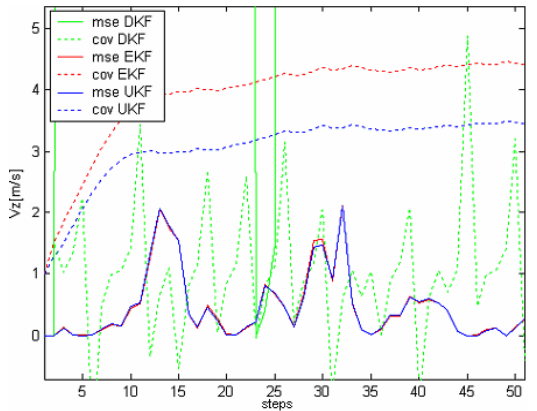

Figure 8: Mean square error of estimated $v_{z}$ and covariance error $P_{v z}$ of speed component. 
786 Computational Methods and Experimental Measurements XIII

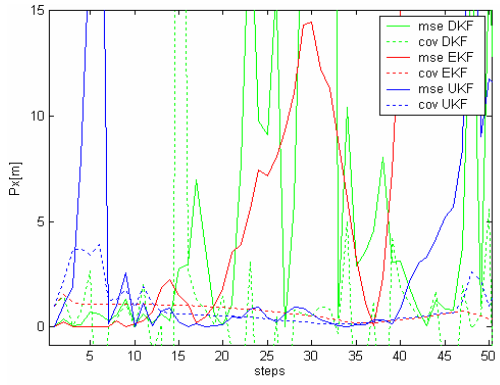

Figure 9: Mean square error of the estimated component $p_{x}$ and covariance error $P_{p x}$ of the position.

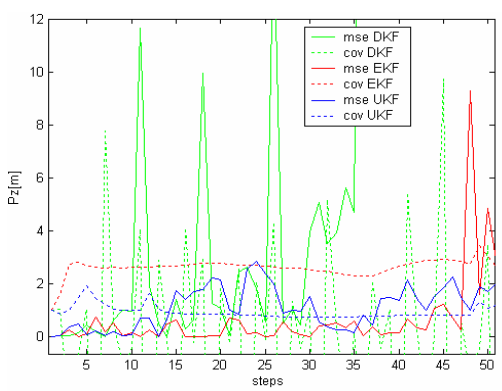

Figure 11: Mean square error of the estimated component $p_{z}$ and covariance error $P_{p z}$ of the position

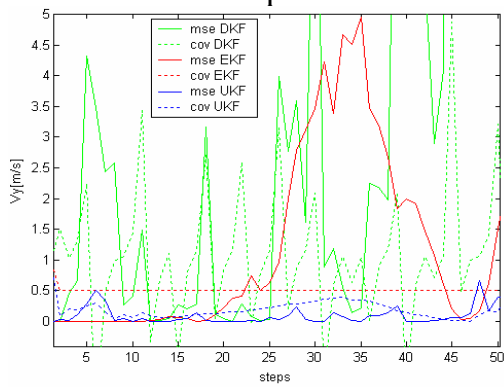

Figure 13: Mean square error of estimated $v_{y} \quad$ and covariance error $P_{v y}$ of speed component.

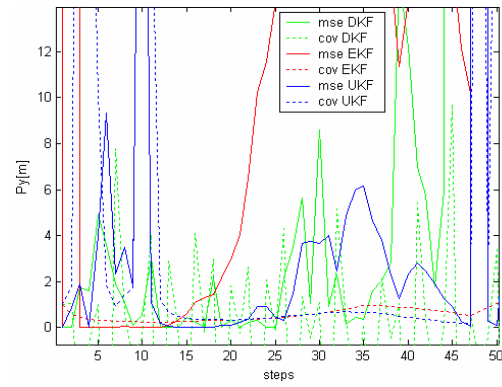

Figure 10: Mean square error of the estimated component $p_{y}$ and covariance error $P_{p y}$ of the position.

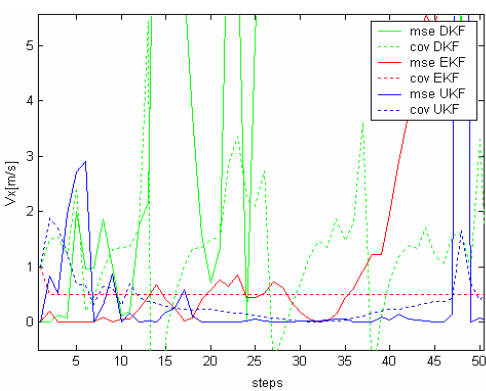

Figure 12: Mean square error of estimated $v_{x}$ and covariance error $P_{v x}$ of speed component.



Figure 14: Mean square error of estimated $v_{z}$ and covariance error $P_{v z}$ of speed component. 
The estimation of object speed in the Cartesian coordinates (Fig. 15-17) and determination of mean square error and covariance error of speed components (Figs. 18-20) has also been done.

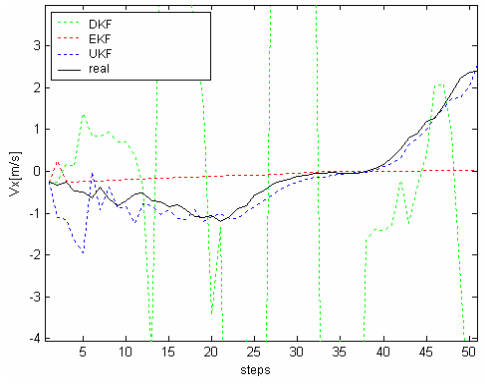

Figure 15: Estimated $v_{x}$ speed component.

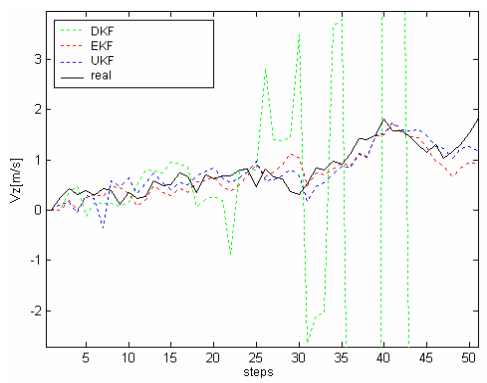

Figure 17: Estimated $v_{z}$ speed component.

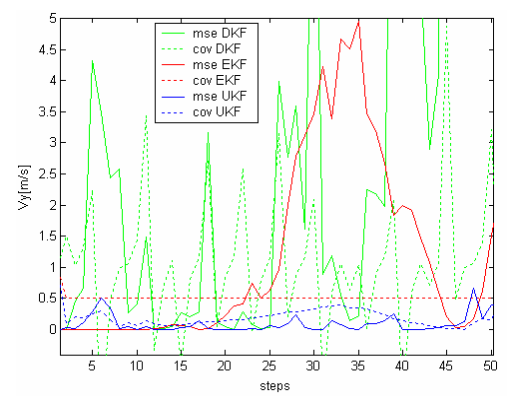

Figure 19: Mean square error of estimated $v_{y}$ and covariance error $P_{v y}$ of speed component.

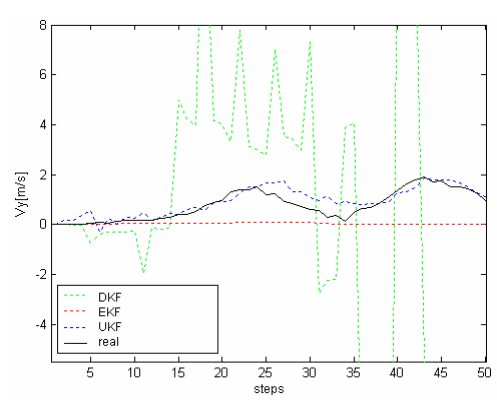

Figure 16: Estimated $v_{y}$ speed component.

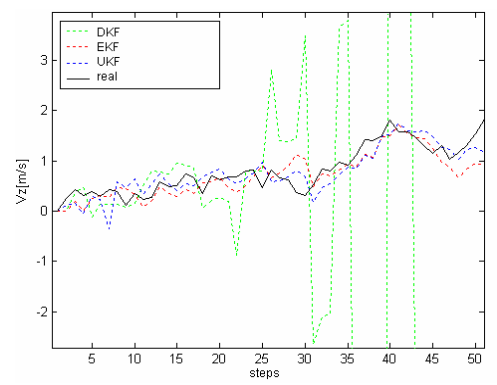

Figure 18: Mean square error of estimated $v_{x}$ and covariance error $P_{v x}$ of speed component.

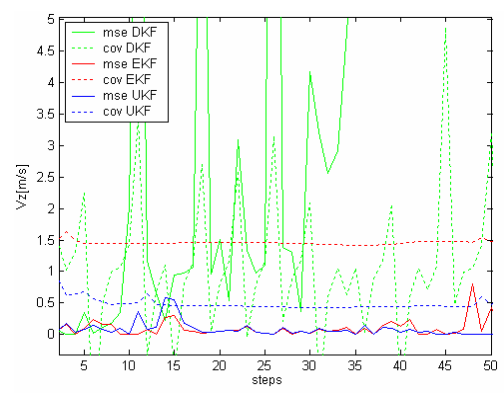

Figure 20: Mean square error of estimated $v_{z}$ and covariance error $P_{v z}$ of speed component. 


\subsection{Analysis of simulation results}

For nonlinear measurement model extended and unscented filters estimate the object position nearly identically. Discrete Kalman filter is becoming nonoptimal filter in the sense of minimizing the mean square error. When the mean square error is included within the area determined by the covariance error of the estimated vector state then filter is performing correctly. Last principle is satisfied for extended and unscented Kalman filters but not for DKF. During speed estimation one can see, that difference between extended and unscented filters is minimal. UKF gives smaller errors what results from nature of speed components, which are band-limited Gaussian processes. The bigger are the jumps of noise values the worse of extended Kalman filter performance is.

\section{Conclusions}

Results of estimation using Discrete and Extended and Unscented Kalman Filter for system with constant velocity and for system with complex movement show that Unscented Kalman Filter operating as algorithm of data processing in system with nonlinear dynamics guarantees the best quality. Nonlinear transform makes Discrete Kalman Filter not to be optimal in sense of minimum mean square error. Loss stabilization in EKF is possible for long measurement steps whereas decreasing of measurement steps enlarges computational costs as a result of complicated calculations of Jacobians. UKF algorithm does not require calculation of Jacobians which simplifies its complexity. The Unscented Kalman Filter provides effective estimation in case of strongly nonlinear models what recommends its use in practice.

\section{References}

[1] Brown R.G., Hwang P.Y.C.: Introduction to Random Signals and Applied Kalman Filtering with MATLAB Exercises and Solutions. John Wiley \& Sons, Canada, 1997.

[2] Gordon N.J., Ristic B., Arulampalam S.: Beyond the Kalman Filter Particle Filters for Tracking Applications. Artech House, London, 2004.

[3] Grewal M.S., Andrews A.P: Kalman filtering Theory and Practice Using MATLAB. John Wiley \& Sons, Canada, 2001.

[4] Julier S.J., Uhlmann J.K., Durrant-Whyte H.F.: A New Method for the Nonlinear Transformation of Means and Covariances in Filters and Estimators. IEEE Transactions on Automatic Control, vol. 45, no. 3, March 2000, pp. 477-482.

[5] van der Merwe R., Wan E.A.: The Square-root Unscented Kalman Filter for State and Parameter-estimation. Proceedings of International Conference on Acoustics, Speech, and Signal Processing, vol. 6, Salt Lake City, May 2001, pp. 3461-3464. 
[6] van der Merwe R., Wan E.A.: The Unscented Kalman Filter. Department of Electrical and Computer Engineering, Oregon Graduate Institute of Science and Technology, Beaverton, Oregon, 2001.

[7] Wan E.A., van der Merwe R.: The Unscented Kalman Filter to appear in Kalman Filtering and Neural Networks. Chapter 7. Edited by Simon Haykin, John Wiley \& Sons, USA, 2001.

[8] Wan E.A., van der Merwe R.: The Unscented Kalman Filter for Nonlinear Estimation. Proc. IEEE Symp. Adaptive Systems for Signal Proc., Communication and Control (AS-SPCC), Lake Louise, Alberta, Canada, October 2000, pp. 153-158. 